\title{
Microscopic description of the pygmy dipole resonance in neutron-rich $\mathrm{Ca}$ isotopes
}

\author{
N. N. Arsenyev ${ }^{1, *}, A . P$. Severyukhin ${ }^{1,2}, V . V$. Voronov $^{1}$, and $N . V$. Giai $^{3}$ \\ ${ }^{1}$ Bogoliubov Laboratory of Theoretical Physics, Joint Institute for Nuclear Research, 141980 Dubna, Moscow region, Russia \\ ${ }^{2}$ Dubna State University, 141982 Dubna, Moscow region, Russia \\ ${ }^{3}$ Institut de Physique Nucléaire, CNRS-IN2P3, Université Paris-Sud, F-91406 Orsay Cedex, France
}

\begin{abstract}
We study the effects of the phonon-phonon coupling on the low-energy electric dipole response within a microscopic model based on an effective Skyrme interaction. The finite rank separable approach for the quasiparticle random phase approximation is used. Choosing as an example the isotopic chain of Calcium, we show the ability of the method to describe the low-energy $E 1$ strength distribution. With one and the same set of parameters we describe available experimental data for ${ }^{48} \mathrm{Ca}$ and predict the electric dipole strength function for ${ }^{50} \mathrm{Ca}$.
\end{abstract}

\section{Introduction}

With the fast development of radioactive beam techniques over the past several decades, experiments focused on the exotic short-lived nuclei were performed extensively. New interesting phenomena arise in nuclei with a strong imbalance of the proton (p) and neutron (n) numbers [1]. One of the phenomena sensitive to the change in $N / Z$ ratios is the pygmy dipole resonance (PDR). The PDR leads to the enhancement of dipole strength well below the region of the giant dipole resonance (GDR). In analogy to the GDR, the PDR has been interpreted as a collective oscillation of the neutron skin, $\Delta R_{\mathrm{np}}$, with respect to a $N \approx Z$ inert core; see, e.g., Refs. $[2,3]$. The total sum of the measured energyweighted sum rule (EWSR) of this $E 1$ distributions is less than $1 \div 2 \%$ of the Thomas-Reiche-Kuhn (TRK) sum rule value for stable nuclei and less than $5 \div 6 \%$ for unstable neutron-rich nuclei [1]. Nevertheless, currently the structure and dynamics of the PDR is one of the actively studied topics in nuclear physics. One of the reason is that the PDR study is expected to provide information on the symmetry energy term of the nuclear equation of state [3].

The study of new exotic nuclei (or/and modes of excitation) stimulates the development of nuclear models to describe properties of nuclei away from the stability valley. The quasiparticle random phase approximation (QRPA) with a self-consistent mean-field derived from Skyrme energy density functionals (EDF) is one of the most successful methods for studying the low-energy dipole strength, see e.g., Refs. [2, 3]. A description of the low-energy $E 1$ strength distribution requires to include the coupling between one- and two-phonon components of the wave functions $[4,5]$. The main difficulty is that the complexity of calculations beyond standard QRPA increases rapidly with the size of the configuration space, so that

\footnotetext{
*e-mail: arsenev@theor.jinr.ru
}

one has to work within limited spaces. With the finite rank separable approximation (FRSA) [6, 7] for the residual interaction, one can perform Skyrme-QRPA calculations in very large two-quasiparticle $(2 \mathrm{QP})$ spaces. Following the basic ideas of the quasiparticle-phonon model (QPM) [8], the FRSA has been generalized for the phonon-phonon coupling (PPC) [9].

The FRSA was used while studing the electric lowenergy excitations and giant resonances within and beyond the Skyrme-QRPA approach [9-11]. In this paper, we discuss the PPC effect on the properties of PDR in the $\mathrm{Ca}$ isotopes. We illustrate our approach with the stable isotope ${ }^{48} \mathrm{Ca}$ having the closed neutron shell $N=28$, in comparison with the unstable isotope ${ }^{50} \mathrm{Ca}$ with $N=30$. These nuclides from the $\mathrm{Ca}$ chain are suitable candidates to follow the PDR evolution. Our results for neutron-rich $\mathrm{Ca}$ and $\mathrm{Sn}$ isotopes were reported in Refs. [12-14].

\section{Brief outline of the FRSA model}

The FRSA approach has been discussed in detail in Refs. $[6,7,14]$ and we briefly present it here for completeness. The SLy5 [15] and SLy5+T [16] EDF are used in the Hartree-Fock-BCS (HF-BCS) calculations as well as for the particle-hole (p-h) channel. The parameters of the Skyrme force SLy5 have been adjusted to reproduce nuclear matter properties, as well as nuclear charge radii and binding energies of doubly magic nuclei. The force SLy5+T involves tensor terms added without refitting the parameters of the central interaction (the tensor interaction parameters are $\alpha_{T}=-170 \mathrm{MeVfm}^{5}$ and $\beta_{T}=100 \mathrm{MeVfm}^{5}$ ). These parameterizations enable to correctly describe binding energies of even-even Ca isotopes. This is illustrated in Fig. 1, where the calculated binding energies for ${ }^{40-60} \mathrm{Ca}$ together with experimental and extrapolated data (AME2016) [17] are shown. The agree- 


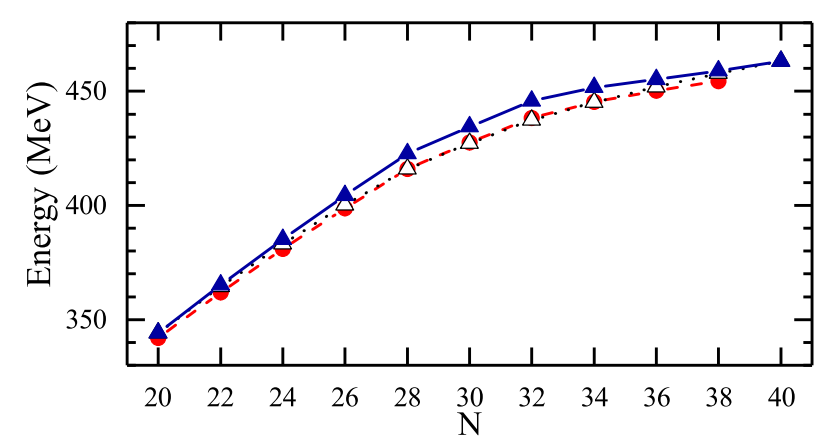

Figure 1. (Color online) Binding energies of the even-even Ca isotopes as a function of neutron number. Results of calculations within the HF-BCS with the SLy5 EDF - open triangles, with SLy5+T EDF - filled triangles. Experimental and extrapolated energies from the AME2016 atomic mass evaluation [17] - filled circles.

ment between the HF-BCS results and data is reasonable, the deviations being less than $2 \%$. For the interaction in the particle-particle (p-p) channel, we use a zerorange volume force. The pairing strength is taken equal to $-270 \mathrm{MeVfm}^{3}$. This value is fitted to reproduce the experimental neutron pairing gaps of ${ }^{50,52,54} \mathrm{Ca}$ obtained by the three-point formula [7, 14]. This kind of pairing interaction has allowed describe satisfactorily experimental data for ${ }^{70,72,74,76} \mathrm{Ni}[18],{ }^{90,92} \mathrm{Zr}$ and ${ }^{92,94} \mathrm{Mo}$ [10]. Thus, hereafter we use the Skyrme interaction SLy5 with and without tensor components in the particle-hole channel together with the volume zero-range force acting in the $p-p$ channel.

The residual interaction in the p-h channel $V_{\mathrm{res}}^{(\mathrm{p}-\mathrm{h})}$ and in the p-p channel $V_{\text {res }}^{(\mathrm{p}-\mathrm{p})}$ can be obtained as the second derivative of the energy density functional with respect to the particle density and the pair density, accordingly. Following Ref. [6] we simplify $V_{\mathrm{res}}^{(\mathrm{p}-\mathrm{h})}$ by approximating it by the Landau-Migdal form. Moreover, we neglect the $l=1$ Landau parameters (in the case of the Skyrme EDFs, the Landau parameters with $l>1$ are equal to zero). The Landau parameters $F_{0}, G_{0}, F_{0}^{\prime}, G_{0}^{\prime}$ expressed in terms of the Skyrme force parameters depend on the Fermi momentum $k_{\mathrm{F}}$ of nuclear matter [19].

To take into account the effects of the PPC we follow the basic QPM ideas [8]. We construct the wave functions of excited states as a linear combination of one- and twophonon configurations

$$
\begin{gathered}
\Psi_{\nu}(\lambda \mu)=\left(\sum_{i} R_{i}(\lambda v) Q_{\lambda \mu i}^{+}\right. \\
\left.+\sum_{\lambda_{1} i_{1} \lambda_{2} i_{2}} P_{\lambda_{2} i_{2}}^{\lambda_{1} i_{1}}(\lambda v)\left[Q_{\lambda_{1} \mu_{1} i_{1}}^{+} Q_{\lambda_{2} \mu_{2} i_{2}}^{+}\right]_{\lambda \mu}\right)|0\rangle,
\end{gathered}
$$

where $|0\rangle$ is the phonon vacuum, $Q_{\lambda \mu i}^{+}$is the phonon creation operator and $v$ labels the excited states. The coefficients $R_{i}(\lambda v), P_{\lambda_{2} i_{2}}^{\lambda_{1} i_{1}}(\lambda v)$ and energies of the excited states $E_{v}$ are determined from the variational principle which leads to a set of linear equations $[9,10]$. The equations have the same form as in the QPM $[4,5,8]$, but the singleparticle spectrum and the parameters of the residual inter-

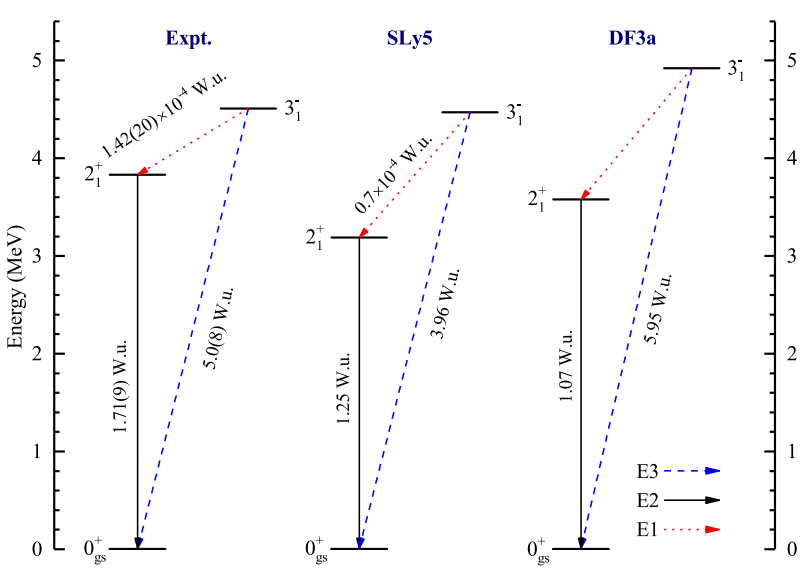

Figure 2. (Color online) Experimental [20] and theoretical energies and transition probabilities of the $\left[2_{1}^{+}\right]_{R P A}$ and $\left[3_{1}^{-}\right]_{R P A}$ states for ${ }^{48} \mathrm{Ca}$. Results for the Skyrme EDF (SLy5) - the present paper, those for the Fayans EDF (DF3a) are taken from [24]. $B(E 1 ; \downarrow), B(E 2 ; \downarrow)$, and $B(E 3 ; \downarrow)$ factors are given in Weisskopf units (W.u.).

action are obtained from the chosen Skyrme EDFs without any further adjustments. In order to let the two-phonon components of the wave functions obey the Pauli principle the exact commutation relations between the phonon operators should be taken into account. In the present case, i.e. for the dipole states which are constructed from $1 \mathrm{p}-\mathrm{h}$ components corresponding to transitions between neighboring main shells the Pauli principle corrections to coupling matrix elements with two-phonon configurations consisting of low-lying phonons of different multipolarities are small (see Ref. [8]).

In order to construct the wave functions (1) of the $1^{-}$states, in the present study we take into account all two-phonon terms built from the phonons with multipolarities $\lambda \leq 5$ [12-14]. As an example the energies and reduced transition probabilities of the first $2^{+}$and $3^{-}$phonons for ${ }^{48,50} \mathrm{Ca}$ are presented in Figs. 2 and 3. The QRPA results obtained with the SLy5 EDF are compared with the experimental data $[20,21]$. The $E 1$ transition matrix elements are calculated with the effective neutron, $e_{(\mathrm{eff})}^{(\mathrm{n})}=-\frac{Z}{A} e$, and proton, $e_{(\mathrm{eff})}^{(\mathrm{p})}=\frac{N}{A} e$, charges. Inclusion of the effective charges eliminates contaminations of the physical response due to the spurious excitation of the center of mass. In Ref. [22], it has been shown that eliminating the spurious state by means of effective charges or the alternative ways (see, e.g., Ref. [23]) leads to very close results. As one can see, the overall agreement of the energies and $B(E \lambda ; \downarrow)$ values with the data looks reasonable. We should be noted that calculations with the forces SLy5 are in good agreement with the values calculated with the DF3a EDF in Ref. [24].

Calculating the electric dipole strength function we include in the model wave function (1) all one-phonon dipole states with energies below $35 \mathrm{MeV}$ and 15 most collective phonons of other multipolarities. The effect of configuration space extension on the results was tested and its minor role was found. 


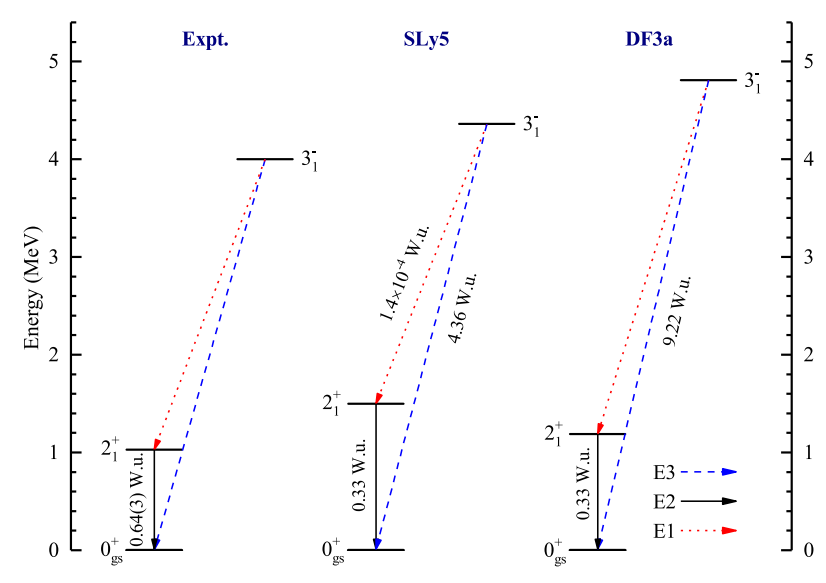

Figure 3. (Color online) The same as in Fig. 2 for ${ }^{50} \mathrm{Ca}$.

\section{Results and discussion}

As the first step, we examine the PPC effect on the $E 1$ strength distributions in ${ }^{48,50} \mathrm{Ca}$ isotopes. It is noteworthy that for all the $E 1$ distributions, considered in the present paper, the matrix elements of direct transitions from the ground state (the phonon vacuum) to two-phonon components are about two orders of magnitude smaller relative to ones for the excitation of one-phonon components [25]. Thus, the transitions from the ground state into the two-phonon part of wave functions (1) are omitted in our analysis. Inspired by Refs. $[14,26]$ we consider as the PDR in ${ }^{48,50} \mathrm{Ca}$ the dipole states placed below $10 \mathrm{MeV}$. Let us now discuss the total value of the $E 1$ strength concentrated in this energy interval. A comparison with recent experimental data $[27,28]$ for ${ }^{48} \mathrm{Ca}$ shows that the RPA approach cannot reproduce the lowenergy part of the $E 1$ strength distribution (see Fig. 4). According to RPA calculations the lowest dipole state has the energy around $10.5 \mathrm{MeV}$. In contrast to the RPA calculations, the inclusion of the two-phonon terms results in the formation of several $1^{-}$states in this energy region (see Fig. 4). The dominant contribution to their wave functions comes from the two-phonon configurations $(>60 \%)$. Their one-phonon parts originate from the fragmentation of the RPA states lying above $10 \mathrm{MeV}$. As one can see in Fig. 4(c), the calculated value of the running sum $\sum B(E 1)$ is close to the experimental value. The PPC calculations give a total value of the dipole strength equals to $0.063 \mathrm{e}^{2} \mathrm{fm}^{2}$ (the summation includes all the dipole states below $10 \mathrm{MeV})$. The experimental value of $\sum B(E 1)$ is $0.0687(75) \mathrm{e}^{2} \mathrm{fm}^{2}$ according to [27] and 0.080(8) $\mathrm{e}^{2} \mathrm{fm}^{2}$ according to [28] for the same interval. Thus, the PPC effects produce a strong impact on the low-energy $E 1$ strength in ${ }^{48} \mathrm{Ca}$. As concerns other theoretical results, the calculations within the relativistic quasiparticle time blocking approximation (RQTBA) estimate the value of $\sum B(E 1)$ as $0.1 \mathrm{e}^{2} \mathrm{fm}^{2}$ [26].

The photon scattering experiments ${ }^{48} \mathrm{Ca}\left(\gamma, \gamma^{\prime}\right)$ allow to determine the sum of energy-weighted $E 1$ strength. According to [27] $0.33(4) \%$ of the TRK sum rule can be attributed to the PDR region of ${ }^{48} \mathrm{Ca}$. The corresponding

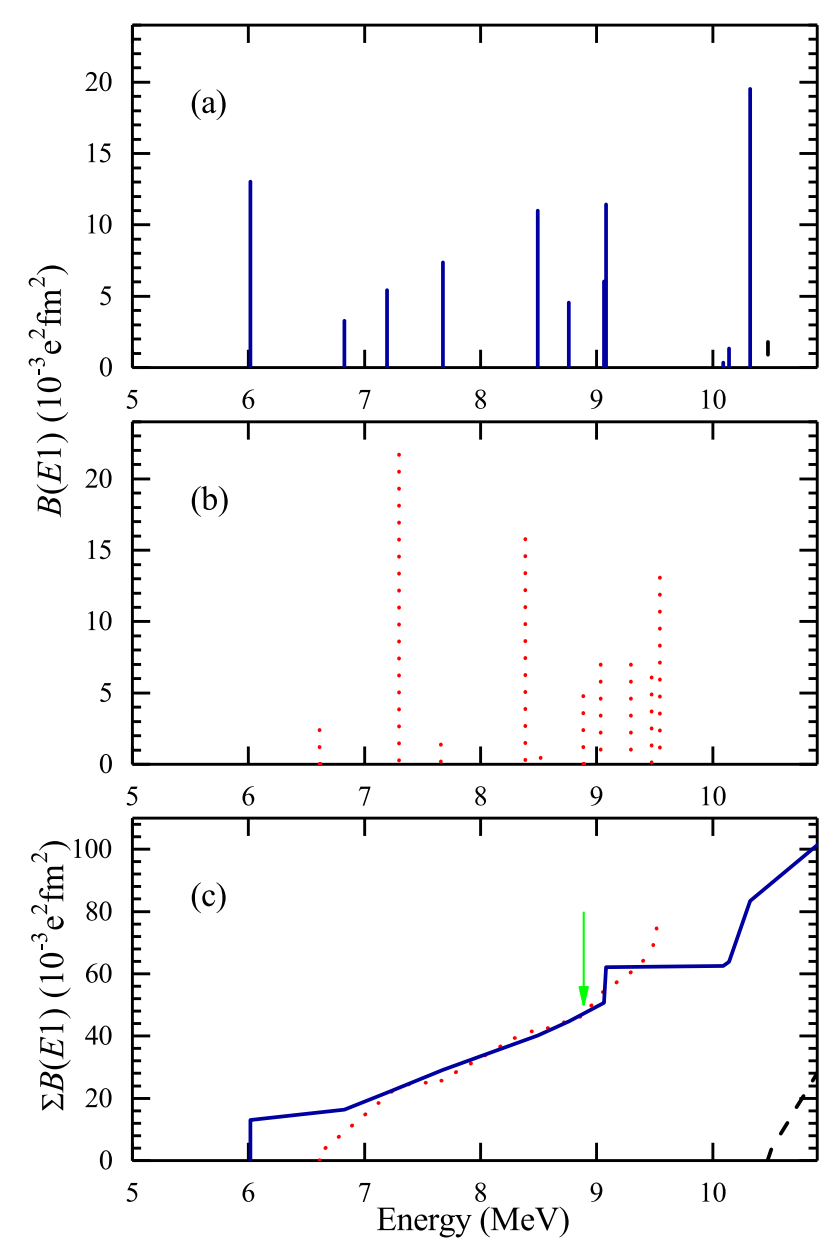

Figure 4. (Color online) $1^{-}$states at $E_{x}<11 \mathrm{MeV}$ in ${ }^{48} \mathrm{Ca}$. Panel (a) $-1^{-}$states calculated within RPA (a small stick in the right corner of the panel) and "RPA+PPC" (solid sticks). Panel (b) - experimental data on electric dipole states from [27, 28]. Panel (c) - calculated (dashed and solid lines) and experimental (dotted line) running sums $\sum B(E 1)$ of electric dipole strength at $E_{x}<11 \mathrm{MeV}$. Calculations are performed with the Skyrme force SLy5 within RPA and "RPA+PPC" aprroaches. The arrow indicates the calculated $S_{1 \mathrm{n}}$ energy.

RQTBA result is $0.55 \%$ [26], whereas our calculations with PPC effects give $0.28 \%$.

Moving from ${ }^{48} \mathrm{Ca}$ to ${ }^{50} \mathrm{Ca}$, the QRPA calculations predict a jump of the $\sum B(E 1)$ value. The neutron number $N=30$ corresponds to the occupation of the neutron $2 p \frac{3}{2}$ subshell, resulting in appearing the two rather pronounced $1^{-}$states below $10 \mathrm{MeV}$. These two states are practically pure neutron 2QP excitations $99 \%\left\{3 s \frac{1}{2} 2 p \frac{3}{2}\right\}_{\mathrm{n}}$ and $98 \%\left\{2 d \frac{5}{2} 2 p \frac{3}{2}\right\}_{\mathrm{n}}$. The contribution from the $2 \mathrm{QP}$ proton components is invisible. As can be seen from Fig. 5, it is these states determine the value of $\sum B(E 1)=0.54 \mathrm{e}^{2} \mathrm{fm}^{2}$.

Thus, the structure of the lowest one-phonon states in ${ }^{50} \mathrm{Ca}$ is very different from that of the lowest $1^{-} \mathrm{RPA}$ state in ${ }^{48} \mathrm{Ca}$. The latter is mainly a proton state built from $2 \mathrm{QP}$ configuration $\left\{2 p \frac{3}{2} 1 d \frac{3}{2}\right\}_{\mathrm{p}}$ giving a contribution of $96 \%$. In ${ }^{48} \mathrm{Ca}$, the closure of the neutron subshell $1 f \frac{7}{2}$ leads to vanishing of the neutron pairing and the $B\left(E 1 ; 0_{g s}^{+} \rightarrow 1_{1}^{-}\right)$ value is exhausted by the proton $2 \mathrm{QP}$ configurations. The 


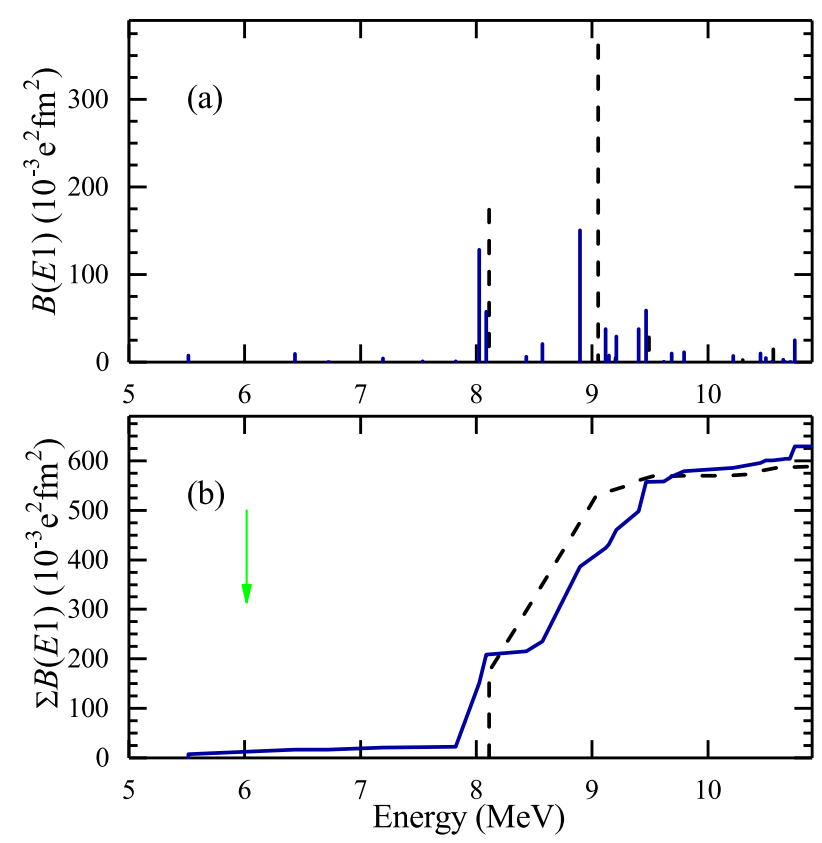

Figure 5. (Color online) Theoretical results for $1^{-}$states at $E_{x}<11 \mathrm{MeV}$ in ${ }^{50} \mathrm{Ca}$. Panel (a) - energies and $B(E 1)$-strengths of $1^{-}$states calculated with the Skyrme force SLy5 within QRPA (dashed sticks) and "RPA+PPC" (solid sticks). Panel (b) - running sums of the dipole strength $\sum B(E 1)$ calculated with the Skyrme force SLy5 within QRPA (dashed line) and "RPA+PPC" (solid line). The arrow indicates the calculated $S_{1 \mathrm{n}}$.

main difference between the two isotopes is that the neutron 2QP configurations contribute more than proton ones in ${ }^{50} \mathrm{Ca}$. This circumstance is mainly responsible for the $\sum B(E 1)$ increase (e.g., Ref. [14]). Calculations with the forces SLy5 and SLy5+T do not change the above conclusion.

The PPC only slightly affect the summed $E 1$ strength below $10 \mathrm{MeV}$ in ${ }^{50} \mathrm{Ca}$ (see the panel (b) in Fig. 5). The phonon-phonon coupling mainly produces the fragmentation of $E 1$ strength among different states, Eq. (1), and the energy shift of dipole spectrum in the low-energy part. The main contribution in the wave function structure of the first $1^{-}$state in ${ }^{50} \mathrm{Ca}$ comes from the two-phonon configuration $\left[2_{1}^{+} \otimes 3_{1}^{-}\right]_{Q R P A}(76 \%)$. This configuration dominates in the structure of the first $1^{-}$state in ${ }^{48} \mathrm{Ca}$ as well.

We found that one-phonon components dominate in the structure of four dipole states in ${ }^{50} \mathrm{Ca}-1_{7}^{-}, 1_{11}^{-}, 1_{13}^{-}$ and $1_{16}^{-}$. The contribution of these components in the norms of the aforementioned states is greater than $86 \%$. These four states give the main contribution to the summed $E 1$ strength below $10 \mathrm{MeV}$. The dominant contribution to the wave functions of other $1^{-}$states comes from the two-phonon configurations ( $>75 \%$ ). We got a total dipole strength of $0.57 \mathrm{e}^{2} \mathrm{fm}^{2}$ for the QRPA and $0.58 \mathrm{e}^{2} \mathrm{fm}^{2}$ for the PPC calculations. The RQTBA result is somewhat less $\left.0.46 \mathrm{e}^{2} \mathrm{fm}^{2}[26]\right)$.

\section{Summary}

Starting from the Skyrme mean-field calculations and QRPA, the distributions of the electric dipole strength in
${ }^{48,50} \mathrm{Ca}$ were studied by taking into account the coupling between one- and two-phonons terms in the wave functions of excited states. The finite-rank separable approach for the QRPA calculations enables one to reduce remarkably the dimensions of the matrices that must be inverted to perform nuclear structure calculations in very large configuration spaces.

Neutron excess effects on the PDR excitation energies and transition strengths were investigated. The impact of the shell closure $N=28$ on the summed $E 1$ strength below $10 \mathrm{MeV}$ was found. The summed dipole transition strength $\sum B(E 1)$ in the PDR region noticeably increases after the crossing the neutron shell $N=28$. At the same time the one-neutron separation energy decreases by $64 \%$ in ${ }^{50} \mathrm{Ca}$ in compare with ${ }^{48} \mathrm{Ca}$. The latter is a result of the pairing effect on neutron $2 p \frac{3}{2}$ subshell for ${ }^{50} \mathrm{Ca}$.

The present model can be extended by complicating the trial model function of dipole states by adding threephonon configurations. The computational developments that would allow us to conclude on this point are underway.

N.N.A., A.P.S., and V.V.V. thank the hospitality of INP-Orsay where a part of this work was done. This work was partly supported by the CNRS-RFBR agreement No. 16-52-150003, the IN2P3-JINR agreement, and the RFBR grant No. 16-02-00228.

\section{References}

[1] D. Savran, T. Aumann, A. Zilges, Prog. Part. Nucl. Phys. 70, 210 (2013)

[2] N. Paar, D. Vretenar, E. Khan, G. Colò, Rep. Prog. Phys. 70, 691 (2007)

[3] X. Roca-Maza, N. Paar, Prog. Part. Nucl. Phys. 101, 96 (2018)

[4] V. G. Soloviev, Ch. Stoyanov, V. V. Voronov, Nucl. Phys. A 304, 503 (1978)

[5] M. Grinberg, Ch. Stoyanov, Nucl. Phys. A 573, 231 (1994)

[6] Nguyen Van Giai, Ch. Stoyanov, V. V. Voronov, Phys. Rev. C 57, 1204 (1998)

[7] A. P. Severyukhin, V. V. Voronov, Nguyen Van Giai, Phys. Rev. C 77, 024322 (2008)

[8] V. G. Soloviev, Theory of Atomic Nuclei: Quasiparticles and Phonons (Institute of Physics, Bristol and Philadelphia, 1992)

[9] A. P. Severyukhin, V. V. Voronov, Nguyen Van Giai, Eur. Phys. J. A 22, 397 (2004)

[10] A. P. Severyukhin, N. N. Arsenyev, N. Pietralla, Phys. Rev. C 86, 024311 (2012)

[11] A. P. Severyukhin, S. Åberg, N. N. Arsenyev, R. G. Nazmitdinov, Phys. Rev. C 95, 061305(R) (2017)

[12] N. N. Arsenyev, A. P. Severyukhin, V. V. Voronov, Nguyen Van Giai, EPJ Web of Conf. 38, 17002 (2012); 107, 05006 (2016)

[13] N. N. Arsenyev, A. P. Severyukhin, V. V. Voronov, Nguyen Van Giai, Acta Phys. Pol. B 46, 517 (2015); ibid. 48, 553 (2017)

[14] N. N. Arsenyev, A. P. Severyukhin, V. V. Voronov, Nguyen Van Giai, Phys. Rev. C 95, 054312 (2017) 
[15] E. Chabanat, P. Bonche, P. Haensel, J. Meyer, R. Schaeffer, Nucl. Phys. A 635, 231 (1998)

[16] G. Colò, H. Sagawa, S. Fracasso, P. F. Bortignon, Phys. Lett. B 646, 227 (2007); ibid. 668, 457(E) (2008)

[17] M. Wang, G. Audi, F. G. Kondev, W. J. Huang, S. Naimi, X. Xu, Chin. Phys. C 41, 030003 (2017)

[18] A. P. Severyukhin, V. V. Voronov, I. N. Borzov, N. N. Arsenyev, Nguyen Van Giai, Phys. Rev. C 90, 044320 (2014)

[19] Nguyen Van Giai, H. Sagawa, Phys. Lett. B 106, 379 (1981)

[20] T. W. Burrows, Nucl. Data Sheets 107, 1747 (2006)

[21] Z. Elekes, J. Timar, B. Singh, Nucl. Data Sheets 112, 1 (2011)

[22] N. N. Arsenyev, A. P. Severyukhin, Phys. Part. Nucl. Lett. 7, 112 (2010)
[23] G. Colò, Nguyen Van Giai, P. F. Bortignon, M. R. Quaglia, Phys. Let. B 485, 362 (2000)

[24] E. E. Saperstein, I. N. Borzov, S. V. Tolokonnikov, JETP Lett. 104, 218 (2016)

[25] V. V. Voronov, Dao Tien Khoa, V. Yu. Ponomarev Bull. Acad. Sci. USSR, Phys. Ser. 48, 190 (1984)

[26] I. A. Egorova, E. Litvinova, Phys. Rev. C 94, 034322 (2016)

[27] T. Hartmann, M. Babilon, S. Kamerdzhiev, E. Litvinova, D. Savran, S. Volz, A. Zilges, Phys. Rev. Lett. 93, 192501 (2004)

[28] V. Derya, D. Savran, J. Endres, M. N. Harakeh, H. Hergert, J. H. Kelley, P. Papakonstantinou, N. Pietralla, V. Yu. Ponomarev, R. Roth, G. Rusev, A. P. Tonchev, W. Tornow, H. J. Wörtche, A.Zilges, Phys. Lett. B 730, 288 (2014) 